\title{
Promoting oral health practice among patients with diabetes attending primary health care clinics
}

\author{
Abeer Aljaber, Khaled Al-Surimi \\ Ministry of Health. King Saud Bin Abdulaziz University for Health Sciences. Saudi Arabia
}

\begin{abstract}
The oral public health program for patients with diabetes was initiated by Saudi Arabia Ministry of Health (MoH) based on international quality standard to control the severity of oral disease in patients with diabetes through improving the accessibility of patients to dental clinics in primary health care centers (PHCC). This program intends to deliver oral health care $(\mathrm{OHC})$ for each patient with diabetes at least one visit every six months. However, we found that more than $90 \%$ of patients with diabetes that visited prince Mohammed bin Saud PHCC in Riyadh do not get their regular dental check up every six months. We developed a quality improvement project (QIP) using the quality improvement model to activate $\mathrm{MoH}$ oral health program for patients with diabetes visiting prince Mohamed bin Saud PHCC.
\end{abstract}

The aim of our QIP was to increase number of patients with diabetes receiving their regular oral health check up during the PHC visit. The quality team tested two simple improvement ideas. The first idea was having the dentist signature on appointment request. The testing of the first idea led to the second idea, that both physician and dentist should sign the referral form. After running several PDSA cycles to test these interventions ideas, we found the number of patients with diabetes seen in dental clinic had increased dramatically compared with the baseline assessment. We conclude that the idea of signing the referral form by both physician and dentist is a practical and simple strategy to be executed and has a direct impact on the patient clinical flow between clinics.

\section{Problem}

The patients' record at Prince Mohammed bin Saud Primary Health Care Center (PHCC), located in Riyadh city, shows that $94 \%$ of diabetic patients usually do not have a regular dental check up every six months as a standard for National Diabetic and Oral Health programs of Ministry of Health $(\mathrm{MoH})$. The $\mathrm{MoH}$ calls for implementing standard as a regular check up for diabetic patients as proactive strategy to prevent and control the oral diseases and its complications among such vulnerable group of patients at $\mathrm{PHC}$ centers.

\section{Background}

Primary Health Care (PHC) services are a vital component of any health care system. Alama-Ata declaration in 1978 called all countries around the world to implement and adopt the PHC approach as strategy to achieve the Health For All.[1] The main objectives of Primary Health Care Centres (PHCC) are to deliver a comprehensive services including promotive, preventive, and curative health services in the right time for the right patients. Oral Health Care $(\mathrm{OHC})$ is considered as a basic component of PHC services to prevent and control the major cause of inflammatory and complications that may lead to adverse events in managing and controlling of various chronic diseases like heart disease and diabetes.[2] It is well documented in many studies [3-5] that there is a significant association between diabetes and severe periodontitis. Thus, the regular dental visits are very important for patients with diabetes to prevent and control developing the periodontitis among patients with diabetes. Research evidence found that this association is highest among patients aged 12 to 18.[6] Patients with diabetes demonstrate an increase in the prevalence of sever periodontal disease that can affect the control of glycaemic level and cause early teeth loss.[7] It is a two way association where the periodontitis affects the glycaemic control in patients with diabetes, and the severity of periodontitis is correlated with poor controlled glycaemic blood level.[8] Thus, controlling periodontitis by $\mathrm{OHC}$ can improve quality of life of patients with diabetes and reduce the cost of diabetic medical care. It has been reported that the reduction of medical cost in periodontal controlled group compared to active periodontal group led to savings of $\$ 2483.51$ in medical costs per patients with diabetes.[9]

The general department for PHCC in $\mathrm{MOH}$ of Saudi Arabia developed policies and procedures to follow up patients in the chronic diseases clinics. However, these policies, procedures, and guidelines are not implemented as required by health care staff in PHCC. According to MOH Policy N\#.4.7, any patients with diabetes should have a follow up visits to chronic disease clinics in all PHCC in Saudi Arabia. The procedures for implementing this policy are: the physician will assess the patient for the disease control status and the adherence to the plan of care according to the clinical guidelines; then the physician will follow up the regular and annual investigations results based on disease' nature; and then the responsible nurse will follow up with other services that includes dental and oral health, health education, and social services.[10]

However, anecdotal evidence shows that there is lack of proper communication between physician and dentist, and nurses, and it has been noticed that nurses are not reinforced by physician or dentist to follow these assigned clinical guidelines. As a result, we 
ended up seeing most patients with diabetes attending PHCC receiving oral health care when it is emergency case with severe pain or acute dental infection.

\section{Baseline measurement}

Previous data from registry of patients with diabetes regularly visiting our primary health care center and the dental clinics records for a random selected period of eight months showed that only 14 out of 236 patients with diabetes were seen in dental clinic, representing $6 \%$ of total patients with diabetes visiting PHCC (see figure 1).

See supplementary file: ds6167.docx - "base line measures and flow chart of processes "

\section{Design}

The oral health care $(\mathrm{OHC})$ program for patients with diabetes attending $\mathrm{MoH} \mathrm{PHC}$ clinics is not activated due to several reasons, and found the primary reasons are the absence of documentation and the lack of effective coordination in referral process between chronic disease clinics and dental clinic. To address the issue, we developed an improvement project with the following improvement ideas:

1. Patient education about the importance of regular dental visit

2. Implement dentist signature on appointment request

3. Physician-dentist referral form signature

4. Check list form in patient file including all referred clinics

5. Phone call or SMS reminder.

The quality team decided to conduct this quality improvement project to activate the documentation and coordination process between the two clinics using the appointment card as tool for linking the patients with diabetes with dental clinic regular check up and having the patient referral form signed by both physician and dentist. Thus, the two process and system level forced functions interventions are highly expected to activate the communication and coordination of patient flow between chronic disease clinic for patients with diabetes and dental clinic to increase the percentage of patients with diabetes seen in dental clinic.

\section{Strategy}

Several PDSA cycles have been tested as follows:

PDSA cycle 1: The patient will be referred to dental clinic by a physician to be examined by dentist and sign the appointment request for the follow up appointment by dentist, then the appointment office will check if the dentist signature is on appointment card, if not the patient will be sent back to dental clinic. As a result of this intervention, all 41 patients were seen in dental clinic except one patient (the appointment officers were busy with their regular patients), but the patient process flow starts working by sending patients to dental clinic before getting their next follow up appointment.

PDSA cycle 2: This cycle aimed to test the idea of having a referral form between chronic disease and dental clinic which should be signed by both physician and dentist. The patient with chronic disease will be referred to dental clinic by referral form, then the nurse will bring the patient and referral form to dental clinic to be examined by the dentist and sign the referral form and keep it in patient file. The second PDSA cycle confirmed our prediction of seeing most of the patients during the second PDSA cycle period as the number of patients increased by $86 \%$. However, we missed seven patients out of 49 patients; five of them missed because the dentist was on short leave at that day afternoon, but this issue has been fixed administratively. We concluded that the idea of signing the referral form by both the physician and dentist led to smooth flow of patient between two clinics. It was also deemed to be very simple and practical to activate the coordination and communication between the two clinics.

\section{Results}

The project results showed a noticeable improvement in number of patients with diabetes being seen in dental clinic as part of their regular visit of PHCC. The post measurements showed that in total 82 patients with diabetes were seen in dental clinic out of 90 patients with diabetes demonstrating $91 \%$ improvement in dental follow up of patients with diabetes compared with the baseline measurement. From figure 3 we can see that the gap between total patients with diabetes in PHCC and patients with diabetes seen in dental clinic was greatly improved after our improvement project.

Thus the idea of having a referral form signed by both physician and dentist is a practical idea to improve the coordination and communication between the two clinics, and matching patients with diabetes with their regular dental check up during their visit of PHCC. Hence, for the purpose of sustainability of the improvement made, the quality improvement team and dental department had discussed the issue to address the shortage of dentist in clinic to examine the referred patients and the decision was that an official memo/circular was sent to dental clinic; if a dentist was not available for any reason the referred patients will be seen by dental hygienist and near appointment with dentist will be arranged. To increase patients awareness about $\mathrm{OHC}$; dentist and dental hygienist will arrange patient education sessions about the importance of regular dental visits with all referred diabetic patients. The importance of oral health care for diabetic patients will be posted in a storyboard in PHCC.

The project team highly recommend to the $\mathrm{PHHC}$ management to make this change idea permanent as a part of the daily practice of patient flow between chronic diseases clinics and dental clinic at Prince Mohammed bin Saud PHCC. Also, we highly recommend that this improvement idea is replicated at other $\mathrm{MoH} \mathrm{PHC}$ clinics, although staff satisfaction should be evaluated to assess the dentist workload and level of satisfaction with the new intervention.

See supplementary file: ds6252.docx - "PDSA1 \&PDSA2 and a comparison graph between pre and post measures" 


\section{Lessons and limitations}

We learned several lessons, for example including that we should focus on healthcare process and addressing patient flow between different clinics when designing a improvement intervention, making sure that the improvement idea should not have a negative impact on the daily practice and functions of other departments in the organisation.

Furthermore, we learned that the communication, coordination, and documentation between clinics is very important to achieve desirable improvement results and to translate written policy, procedures, and guidelines into practice.

\section{Conclusion}

Fixing the patient flow process is manageable in order to make sure that patients with chronic disease attending $\mathrm{PHC}$ centers and clinics could get their regular dental check up during their primary health care visits if there is a good coordination and communication process in place between different clinic through the appointment office who could play a major role in activating the patient flow of patients between different clinics. This simple idea of change will lead practically to implement $\mathrm{MoH}$ standard and polices regarding patients with diabetes should have a regular oral health check up every six months to prevent the severity of periodontitis and diabetes complication.

\section{References}

1. Al-Sakkak MA, Al-Nowaiser NA, Al-Khashan HI, AlAbdrabulnabi AA, Jaber RM. Patient satisfaction with primary health care services in Riyadh. Saudi Med J. 2008;29(3):432-6.

2. Health UDo, Services H. Oral health in America: a report of the surgeon general. Rockville, MD: National Institutes of Health 2000

3. Mealey BL, Oates TW. Diabetes mellitus and periodontal diseases. J Periodontol 2006;77(8):1289-303.

4. Tsai C, Hayes C, Taylor GW. Glycemic control of type 2 diabetes and severe periodontal disease in the US adult population. Community Dent Oral Epidemiol 2002;30(3):182-92

5. Stoykova M, Musurlieva N, Boyadzhiev D. Risk factors for development of chronic periodontitis in Bulgarian patients (pilot research). Biotechnol Equip 2014;28(6):1150-4.

6. Lalla E, Cheng B, Lal S, Tucker S, Greenberg E, Goland R, et al. Periodontal Changes in Children and Adolescents With Diabetes A case-control study. Diabetes Care 2006;29(2):295-9.

7. Elangovan S, Hertzman-Miller R, Karimbux N, Giddon D. A Framework for Physician-Dentist Collaboration in Diabetes and Periodontitis. Clin Diabetes 2014;32(4):188-92.

8. Dale J, Lindenmeyer A, Lynch E, Sutcliffe P. Oral health: a neglected area of routine diabetes care? Brit J Gen Pract 2014;64(619):103-4.

9. Jeffcoat M, Tanna N, Hedlund C. Does treatment of oral disease reduce the costs of medical care. Medscape 2011.

10. Ministry of Health. Saudi Arabia: policies and procedures of nursing work in primary health care; (cited 2015 September 12).

http://www.moh.gov.sa/depts/NursingDepts/Policiesandproc edures/PrimaryHealthcare/Pages/default.aspx

\section{Declaration of interests}

None declared.

\section{Acknowledgements}

Mr. Ahmed alodainy, manager, Prince Mohamed bin Saud Center. Dr. Razan Alanteet, family medicine specialist.

\section{Ethical approval}

Ethical approval is not necessary for this kind of project because the aim of this project was to bring about improvement by activating an oral health care program for diabetic patients, and it is one of primary health care requirements for preventive health care measures of $\mathrm{MoH}$. 